\title{
On the lambda algebra and Singer's cohomological transfer
}

\author{
By Đặng Võ PHúC \\ Faculty of Education Studies, University of Khanh Hoa, Vietnam
}

\begin{abstract}
Writing $\mathbb{A}$ for the 2-primary Steenrod algebra, which is the algebra of stable natural endomorphisms of the mod 2 cohomology functor on topological spaces. Working at the prime 2, computing the cohomology of $\mathbb{A}$ is an important problem of Algebraic topology, because it is the initial page of the Adams spectral sequence converging to stable homotopy groups of the spheres. A relatively efficient tool to describe this cohomology is the Singer algebraic transfer of rank $n$ in [22], which passes from a certain subquotient of a divided power algebra to the cohomology of $\mathbb{A}$. Singer predicted that this transfer is a monomorphism, but this remains open for $n \geq 4$. This short note is to verify Singer's conjecture in the ranks 4 and 5 and some generic degrees.
\end{abstract}

Key words: Primary cohomology operations; Steenrod algebra; Hit problem; Cohomology of Steenrod algebra; Algebraic transfer; Lambda algebra; Adams spectral sequence.

1. Introduction It is well-known that there is a group homomorphism $S q^{n}$ for $n \geq 0$, between mod-2 cohomology groups of a topological space, called Steenrod squares of degrees $n$. They are stable cohomology operations, that is, they commute with suspension maps. All the Steenrod squares form an algebraic structure which is known as the 2primary Steenrod algebra $\mathbb{A}$ subject to the Adem relations. It was applied to the vector fields on spheres and the Hopf invariant one problem, which asks for which $n$ there exist maps of Hopf invariant \pm 1 . So, the Steenrod algebra is one of the important tools in Algebraic topology. Specifically, its cohomology $\operatorname{Ext}_{A}^{*, *}(\mathbb{Z} / 2, \mathbb{Z} / 2)$ is an algebraic object that serves as the input to the Adams spectral sequence (the ASS) [1] and therefore, computing this cohomology is of fundamental importance to the study of the stable homotopy groups of spheres.

The cohomological transfer, defined by Singer [22], could be an useful approach to describe the mysterious structure of the cohomology algebra of A. In order to better understand this transfer, we will use the following notations and the relevant concepts. Let denote $V^{\oplus n}$ the $n$-dimensional vector space over the prime field $\mathbb{Z} / 2$. Then, we write $H^{*}\left(V^{\oplus n}\right)$ and $H_{*}\left(V^{\oplus n}\right)$ for mod-2 cohomology and homology of $B V^{\oplus n}$ (the classifying space of $V^{\oplus n}$ ). One should note that $B V^{\oplus n}$ is homotopy equivalent to the cartesian product of $n$ copies of the union of the finite projective spaces. As it is known, $H^{*}\left(V^{\oplus n}\right)$ is identified with the polynomial algebra $\mathbb{Z} / 2\left[u_{1}, \ldots, u_{n}\right]$ on generators of degree 1 , equipped with the canonical unstable algebra structure over the Steenrod algebra (i.e., it is a commutative, associative, graded $\mathbb{Z} / 2$-algebra equipped with a structure of unstable $\mathbb{A}$-module and satisfying two relations, one called the Cartan formula, and the other called the instability relation: $S q^{\operatorname{deg}(x)}(x)=$ $x^{2}$.) By dualizing, $H_{*}\left(V^{\oplus n}\right)$ has a natural basis dual to the monomial basis of $H^{*}\left(V^{\oplus n}\right)$. We denote by $a_{1}, \ldots, a_{n}$ the basis of $H_{1}\left(V^{\oplus n}\right)$ dual to the basis $u_{1}, \ldots, u_{n}$ of $H^{1}\left(V^{\oplus n}\right)=\operatorname{Hom}\left(V^{\oplus n}, \mathbb{Z} / 2\right)$, so that $a_{i}\left(u_{j}\right)=1$ if $i=j$ and is 0 if $i \neq j$. We write the dual of $u_{1}^{d_{1}} \ldots u_{n}^{d_{n}}$ as $a_{1}^{\left(d_{1}\right)} \ldots a_{n}^{\left(d_{n}\right)}$ where the parenthesized exponents are called divided powers, and be careful that in the corresponding situation over a field of characteristic 0 in place of $\mathbb{Z} / 2, a_{i}^{\left(d_{i}\right)}=a_{i}^{d} / d$ !, which fits with the formula $a^{(d)} a^{(e)}=\left(\begin{array}{c}d+e \\ d\end{array}\right) a^{(d+e)}$. This product gives a commutative graded algebra $\Gamma\left(a_{1}, \ldots, a_{n}\right)$ over $\mathbb{Z} / 2$ called a divided power algebra, where $\Gamma\left(a_{1}, \ldots, a_{n}\right)=H_{*}\left(V^{\oplus n}\right)$, and an element $a_{1}^{\left(d_{1}\right)} \ldots a_{n}^{\left(d_{n}\right)}$ in $H_{*}\left(V^{\oplus n}\right)$ corresponding to a monomial $u_{1}^{d_{1}} \ldots u_{n}^{d_{n}}$ in $H^{*}\left(V^{\oplus n}\right)$ is called $d$-monomial. Now, let $P_{\mathbb{A}} H_{*}\left(V^{\oplus n}\right)$ be the subspace of $H_{*}\left(V^{\oplus n}\right)$ consisting of all elements that are annihilated by all Steenrod squares of positive degrees. The general linear group $G L_{n}=G L\left(V^{\oplus n}\right)$ acts regularly on the classifying space $B V^{\oplus n}$ and 
therefore on $H^{*}\left(V^{\oplus n}\right)$ and $H_{*}\left(V^{\oplus n}\right)$. This action commutes with that of the algebra $\mathbb{A}$ and so acts $\mathbb{Z} / 2 \otimes_{\mathbb{A}} H^{*}\left(V^{\oplus n}\right)$ and $P_{\mathbb{A}} H_{*}\left(V^{\oplus n}\right)$. For each $n \geq 0$, Singer constructed in [22] a linear transformation from $P_{\mathbb{A}} H_{*}\left(V^{\oplus n}\right)$ to the $n$-th cohomology group $\operatorname{Ext}_{\mathbb{A}}^{n, n+*}(\mathbb{Z} / 2, \mathbb{Z} / 2)$ of $\mathbb{A}$, which commutes with two $S q^{0}$ 's on $P_{\mathbb{A}} H_{*}\left(V^{\oplus n}\right)$ and $\operatorname{Ext}_{\mathbb{A}}^{n, n+*}(\mathbb{Z} / 2, \mathbb{Z} / 2$ ) (see Boardman [2] and Minami [13] for more about this). He shows that this map factors through the quotient of its domain's $G L_{n}$-coinvariants to give rise the so-called cohomological transfer of rank $n$ $\phi_{n}^{*}(\mathbb{Z} / 2): \mathbb{Z} / 2 \otimes_{G L_{n}} P_{\mathbb{A}} H_{*}\left(V^{\oplus n}\right)$ $\longrightarrow \operatorname{Ext}_{\mathbb{A}}^{n, n+*}(\mathbb{Z} / 2, \mathbb{Z} / 2)$

The domain of this transfer is dual to the space of $G L_{n}$-invariants $\left(\mathbb{Z} / 2 \otimes_{\mathbb{A}} H^{*}\left(V^{\oplus n}\right)\right)^{G L_{n}}$. It is to be noted that $\phi_{n}^{*}(\mathbb{Z} / 2)$ is induced over the $E_{2}$-term of the ASS by the geometrical transfer map $\Sigma^{\infty}\left(B\left(V^{\oplus n}\right)_{+}\right) \longrightarrow \Sigma^{\infty}\left(\mathbb{S}^{0}\right)$ in stable homotopy theory (see also Mitchell [14]). The work of Minami [13] indicated that these transfers play a key role in finding permanent cycles in the ASS. In the second cohomology groups of $\mathbb{A}$, following Mahowald [11] and Lin-Mahowald [9], the classes $h_{1} h_{j}$ for $j \geq 3$ and $h_{j}^{2}$ for $0 \leq j \leq 5$, are known to be the permanent cycles in the ASS. In 2016, Hill, Hopkins, and Ravenel [6] showed that when $j \geq 7$, the class $h_{j}^{2}$ is not a permanent cycle in the ASS. It is surprising that so far there is no answer for $j=6$. The question of whether these $h_{j}^{2}$ are the permanent cycles in the ASS or not is called Kervaire invariant problem in literature [4]. This is one of the oldest unresolved issues in Differential and Algebraic topology.

Direct calculating the value of $\phi_{n}^{*}(\mathbb{Z} / 2)$ on any non-zero element is a hard work. It has been demonstrated that $\phi_{n}^{*}(\mathbb{Z} / 2)$ is an isomorphism for $n \leq 2$ by Singer himself [22], and $n=3$ by Boardman [2]. Most notably, Singer sets up a hypothesis in the same paper [22] that $\phi_{n}^{*}(\mathbb{Z} / 2)$ is a monomorphism, but this is still not confirmed, for all cohomological degrees $n \geq 4$. The cases $n=4,5$ are our concern in this paper. Besides Singer's transfer homomorphism, the lambda algebra $\Lambda$ of Bousfield et al. [3] is also a relatively efficient tool to compute the cohomology of the Steenrod algebra. Recall that $\Lambda$ is the quotient of the graded tensor algebra over $\mathbb{Z} / 2$ on symbols $\lambda_{i}$ for $i \geq-1$, modulo the two-sided ideal generated by $\lambda_{s} \lambda_{k}-\sum_{j}\left(\begin{array}{c}j-k-1 \\ 2 j-s\end{array}\right) \lambda_{s+k-j} \lambda_{j}$, for any $s, k \geq-1$ by the right ideal generated by $\lambda_{-1}$. An interesting representation in the algebra $\Lambda$ of the algebraic transfer, established by Chơn and Hà [5], is a $\mathbb{Z} / 2$-linear map $\psi_{n}$ from $P_{\mathbb{A}} H_{*}\left(V^{\oplus n}\right)$ to a subspace of $\Lambda$ spanned by all monomials of length $n$ in all the monomials in $\lambda_{i}$. The authors showed that the image of an element $\zeta \in P_{\mathbb{A}} H_{*}\left(V^{\oplus n}\right)$ under $\psi_{n}$ is a cycle in $\Lambda$ and $\left[\psi_{n}(\zeta)\right]=\phi_{n}^{*}(\mathbb{Z} / 2)([\zeta])$. Note also that this result is a dual version of the one in Hưng [7].

The Singer transfer we are discussing is closely related to the hit problem in literature [16] of determination of a minimal generating set for the unstable $\mathbb{A}$-module $H^{*}\left(V^{\oplus n}\right)$. The reader can find an excellent list of publications about this problem in the works by Kameko [8], Mothebe-Uys [15], the present writer [17, 18, 21], Singer [23], Sum [24, 26], WalkerWood [28], Wood [29] and others. Hit problems are motivated by several problems in Topology and Algebra. It was completely studied for the cases $n \leq 4$ by Peterson [16], Kameko's thesis [8] and Sum [24]. Nevertheless, the general answer seems to be out of reach with the present techniques. Therefore, it is renowned as a difficult problem, even with the help of a computer. In fact, when $\mathbb{Z} / 2$ is a trivial $\mathbb{A}$ module, solving the hit problem is equivalent to determining the "cohits" $\mathbb{Z} / 2 \otimes_{\mathbb{A}} H^{*}\left(V^{\oplus n}\right)$ as a graded vector space, or more generally as a graded module over the group algebra $\mathbb{Z} / 2\left[G L_{n}\right]$. Frank Peterson [16] conjectured that $\mathbb{Z} / 2 \otimes_{\mathbb{A}} H^{*}\left(V^{\oplus n}\right)=0$ unless $\alpha(n+*) \leq n$, where $\alpha(k)$ is the number of 1 's in the dyadic expansion of a positive integer $k$. His motivation for this was to prove that if $\mathcal{M}$ is a smooth manifold of dimension $*$ such that all products of length $n$ of Stiefel-Whiney classes of its nomal bundle vanish, then either $\alpha(*) \leq n$ or $\mathcal{M}$ is cobordant to zero. The conjecture was established by Wood [29]. Therefrom, to study $\mathbb{Z} / 2 \otimes_{\mathbb{A}} H^{*}\left(V^{\oplus n}\right)$ in each $n$ and degree $* \geq 0$, it suffices by Peterson's conjecture and iteration of the Kameko map [8] to consider degrees $*$ in the following "generic" form:

(1) $k\left(2^{t}-1\right)+\ell .2^{t}$, for $k, t, \ell \geq 0, \mu(\ell)<k \leq n$,

where $\mu(\ell)$ is a smallest number $r \in \mathbb{N}$ such that $\alpha(\ell+r) \leq r$. By Minami [12], hit problems are also considered as an useful tool for studying permanent cycles in the ASS.

In the present work, we explicitly determine the structure of the coinvariant $\mathbb{Z} / 2 \otimes_{G L_{n}} P_{\mathbb{A}} H_{*}\left(V^{\oplus n}\right)$ and the behavior of the cohomological transfer of ranks 4 and 5 in some generic degrees of the form (1) by using techniques of the hit problem and the representation in the algebra $\Lambda$ of these transfers. 
2. Main results To begin with, we remark that by Sum [24], it is enough to depict the behavior of the fourth transfer in the following degrees $d$ :

(i) $d=2^{t+1}-r$, for $1 \leq r \leq 3$,

(ii) $d=2^{t+s+1}+2^{t+1}-3$,

(iii) $d=2^{t+s}+2^{t}-2$,

(iv) $d=2^{t+s+u}+2^{t+s}+2^{t}-3$,

whenever $r, t, s$, and $u$ are positive integers. It is not difficult to check that the above degrees can be rewritten as (1). The cases of (i) are known by Sum [25]. The results for (ii) and (iii) have been partially probed in $[19,20]$. This note is to investigate the case (iv).

Now, let us state the main results of this text. We first study the fourth cohomological transfer in degrees $d_{t, s, u}:=2^{t+s+u}+2^{t+s}+2^{t}-3$. To make this, we give an explicit description of a minimal generating set for the domain of $\phi_{4}^{*}(\mathbb{Z} / 2)$ in degree $d_{t, s, u}$, and obtain the following, which is proved in many steps by using computational techniques of the hit problem of four variables and some previous results by the present author [19, 20] and Sum [25].

Theorem 2.1. The domain of $\phi_{4}^{*}(\mathbb{Z} / 2)$ in degree $d_{t, s, u}$ is determined by

$$
\begin{aligned}
& \mathbb{Z} / 2 \otimes_{G L_{4}} P_{\mathbb{A}} H_{d_{t, s, u}}\left(V^{\oplus 4}\right) \\
& = \begin{cases}0 & \text { if } s=1, u=1 \text { and } t \geq 1, \\
0 & \text { if } s=2, u=1 \text { and } t \geq 2, \\
0 & \text { if } s \geq 3, u=1 \text { and } t \geq 1, \\
0 & \text { if } s=1, u=2 \text { and } t=1, \\
\left\langle\left[\zeta_{t, 1,2}\right]\right\rangle, & \text { if } s=1, u=2 \text { and } t \geq 2, \\
0 & \text { if } s=1, u \geq 3 \text { and } t \geq 1, \\
\left\langle\left[\zeta_{1,2, u}\right]\right\rangle, & \text { if } s=2, u \geq 1 \text { and } t=1, \\
0 & \text { if } s \geq 3, u \geq 2 \text { and } t=1, \\
\left\langle\left[\zeta_{t, s, u}\right]\right\rangle, & \text { if } s \geq 2, u \geq 2 \text { and } t \geq 2,\end{cases}
\end{aligned}
$$

where

$$
\begin{aligned}
\zeta_{t, 1,2}= & a_{1}^{(0)} a_{2}^{\left(2^{t+2}-1\right)} a_{3}^{\left(2^{t+2}-1\right)} a_{4}^{\left(3.2^{t}-1\right)} \\
& +a_{1}^{(0)} a_{2}^{\left(2^{t+2}-1\right)} a_{3}^{\left(5.2^{t}-1\right)} a_{4}^{\left(2^{t+1}-1\right)} \\
& +a_{1}^{(0)} a_{2}^{\left(6.2^{t}-1\right)} a_{3}^{\left(3.2^{t}-1\right)} a_{4}^{\left(2^{t+1}-1\right)} \\
& +a_{1}^{(0)} a_{2}^{\left(7.2^{t}-1\right)} a_{3}^{\left(2^{t+1}-1\right)} a_{4}^{\left(2^{t+1}-1\right)} \\
\zeta_{1,2, u}= & a_{1}^{\left(2^{u+3}-1\right)} a_{2}^{(3)} a_{3}^{(3)} a_{4}^{(2)} \\
& +a_{1}^{\left(2^{u+3}-1\right)} a_{2}^{(3)} a_{3}^{(4)} a_{4}^{(1)} \\
& +a_{1}^{\left(2^{u+3}-1\right)} a_{2}^{(5)} a_{3}^{(2)} a_{4}^{(1)} \\
& +a_{1}^{\left(2^{u+3}-1\right)} a_{2}^{(6)} a_{3}^{(1)} a_{4}^{(1)} \\
\zeta_{t, s, u}= & a_{1}^{(0)} a_{2}^{\left(2^{t}-1\right)} a_{3}^{\left(2^{s+t}-1\right)} a_{4}^{\left(2^{s+t+u}-1\right)}
\end{aligned}
$$

It would be interesting also to see that by this theorem, we have isomorphisms:

$\mathbb{Z} / 2 \otimes_{G L_{4}} P_{\mathbb{A}} H_{d_{t, 1,2}}\left(V^{\oplus 4}\right) \cong \mathbb{Z} / 2,(t \geq 2)$,

$\mathbb{Z} / 2 \otimes_{G L_{4}} P_{\mathbb{A}} H_{d_{1,2, u}}\left(V^{\oplus 4}\right) \cong \mathbb{Z} / 2,(u \geq 1)$,

$\mathbb{Z} / 2 \otimes_{G L_{4}} P_{\mathbb{A}} H_{d_{t, s, u}}\left(V^{\oplus 4}\right) \cong \mathbb{Z} / 2,(t \geq 2, s \geq 2, u \geq$ 2 ).

Since the elements $\zeta_{t, 1,2}, \quad \zeta_{1,2, u}$ and $\zeta_{t, s, u}$ belong to $P_{\mathbb{A}} H_{d_{t, 1,2}}\left(V^{\oplus 4}\right), \quad P_{\mathbb{A}} H_{d_{1,2, u}}\left(V^{\oplus 4}\right)$ and $P_{\mathbb{A}} H_{d_{t, s, u}}\left(V^{\oplus 4}\right)$, respectively, $\psi_{4}\left(\zeta_{t, 1,2}\right), \psi_{4}\left(\zeta_{1,2, u}\right)$ and $\psi_{4}\left(\zeta_{t, s, u}\right)$ are cycles in the lambda algebra $\Lambda$. Furthermore, using the representation in $\Lambda$ of the fourth transfer map, it may be concluded that

$$
\begin{aligned}
& \phi_{4}^{*}(\mathbb{Z} / 2)\left(\left[\zeta_{t, 1,2}\right]\right)=\left[\psi_{4}\left(\zeta_{t, 1,2}\right)\right] \\
& =\left[\lambda_{0} \lambda_{2^{t+2}-1}^{2} \lambda_{2^{t+1}+2^{t}-1}\right] \\
& =h_{0} c_{t} \in \operatorname{Ext}_{\mathbb{A}}^{4,4+d_{t, 1,2}}(\mathbb{Z} / 2, \mathbb{Z} / 2), \\
& \phi_{4}^{*}(\mathbb{Z} / 2)\left(\left[\zeta_{1,2, u}\right]\right)=\left[\psi_{4}\left(\zeta_{1,2, u}\right)\right] \\
& =\left[\lambda_{2^{u+3}-1} \lambda_{3}^{2} \lambda_{2}\right] \\
& =h_{u+3} c_{0} \in \operatorname{Ext}_{\mathbb{A}}^{4,4+d_{1,2, u}}(\mathbb{Z} / 2, \mathbb{Z} / 2), \\
& \phi_{4}^{*}(\mathbb{Z} / 2)\left(\left[\zeta_{t, s, u}\right]\right)=\left[\psi_{4}\left(\zeta_{t, s, u}\right)\right] \\
& =\left[\lambda_{0} \lambda_{2^{t}-1} \lambda_{2^{s+t}-1} \lambda_{2^{s+t+u}-1}\right] \\
& =h_{0} h_{t} h_{s+t} h_{s+t+u} \in \operatorname{Ext}_{\mathbb{A}}^{4,4+d_{t, s, u}}(\mathbb{Z} / 2, \mathbb{Z} / 2) .
\end{aligned}
$$

These, together with Theorem 2.1 and a fact of the fourth cohomology groups $\operatorname{Ext}_{\mathbb{A}}^{4,4+d_{t, s, u}}(\mathbb{Z} / 2, \mathbb{Z} / 2)$ (see Lin [10]), yields the following.

Corollary 2.2. The algebraic transfer is an isomorphism in bidegree $\left(4,4+d_{t, s, u}\right)$ for all $t, s, u$.

Next, we are going to survey the behavior of the transfer homomorphism of rank 5 in degrees of the form (1) with $k=n=5$ and $\ell=50$. To accomplish this, the domains of $\phi_{5}^{*}(\mathbb{Z} / 2)$ at these degrees are computed as follows.

Theorem 2.3. Let $d_{t}:=5\left(2^{t}-1\right)+50.2^{t}$ with $t$ an arbitrary non-negative integer. Then, the coinvariant spaces $\mathbb{Z} / 2 \otimes_{G L_{5}} P_{\mathbb{A}} H_{d_{t}}\left(V^{\oplus 5}\right)$ are trivial.

The proof of the theorem is quite long and complicated. We give a brief description for this: Firstly, we compute explicitly the monomial bases for the spaces of indecomposable elements $\mathbb{Z} / 2 \otimes_{\mathbb{A}} H^{*}\left(V^{\oplus 5}\right)$ in degrees $d_{t}$. An effective approach, based on the iterated Kameko squaring operation [8] coupled with the results by Mothebe-Uys [15], Tín [27] and some linear transformations in Sum [24], is applied in our computations Additionally, we also used the MAGMA computer algebra to verify the results. In particular, these calculations confirmed Sum's conjecture [26] for the relationship between $\mathbb{A}$-generators of the algebras $H^{*}\left(V^{\oplus 4}\right)$ and $H^{*}\left(V^{\oplus 5}\right)$ in the above degrees. Next, using those monomial bases, we may claim that the invariants $\left(\mathbb{Z} / 2 \otimes_{\mathbb{A}} H^{*}\left(V^{\oplus 5}\right)\right)^{G L_{5}}$ are 
trivial in degrees $d_{t}$, from which the theorem follows from the fact that $\mathbb{Z} / 2 \otimes_{G L_{5}} P_{\mathbb{A}} H_{*}\left(V^{\oplus 5}\right)$ is isomorphic to $\left(\mathbb{Z} / 2 \otimes_{\mathbb{A}} H^{*}\left(V^{\oplus 5}\right)\right)^{G L_{5}}$.

Now according to [10], it is easily seen that $\operatorname{Ext}_{\mathbb{A}}^{5,5+d_{t}}(\mathbb{Z} / 2, \mathbb{Z} / 2)=\left\langle h_{0} h_{t+1} h_{t+2} h_{t+4} h_{t+5}\right\rangle=0$, for arbitrary $t \geq 0$, and so, by Theorem 2.3, we immediately obtain

Corollary 2.4. The Singer transfer is a trivial isomorphism in bidegree $\left(5,5+d_{t}\right)$ for every nonnegative integer $t$.

Thus, Corollaries 2.2 and 2.4 favor the Singer conjecture in bidegrees $\left(4,4+d_{t, s, u}\right)$ and $\left(5,5+d_{t}\right)$ for any $t, s, u$.

Detailed proofs of all the results of this note will be published elsewhere.

3. Conclusion Although our work does not apparently lead to either a proof or a refutation of the Singer conjecture in general, we feel that it represents an interesting note about an application of hit problem and the lambda algebra for studying this conjecture. Perhaps a continuation of our methods may provefruitful. It is our belief that further research canspring from these ideas.

\section{References}

[ 1 ] J.F. Adams, On the non-existence of elements of Hopf invariant one, Ann. of Math. (2) 72 (1960), 20-104.

[ 2 ] J. M. Boardman, Modular representations on the homology of power of real projective space, in: M. C. Tangora (Ed.), Algebraic Topology, Oaxtepec, 1991, in: Contemp. Math. vol. 146, 1993, pp. 4970.

[ 3 ] A.K. Bousfield, E.B. Curtis, D.M. Kan, D.G. Quillen, D.L. Rector and J.W. Schlesinger, The mod-p lower central series and the Adams spectral sequence, Topology 5 (1966), 331-342.

[4] W. Browder, The Kervaire invariant of framed manifolds and its generalization, Ann. of Math. (2) 90 (1969), 157-186.

[ 5 ] P.H. Chơn and L.M. Hà , Lambda algebra and the Singer transfer, C. R. Math. Acad. Sci. Paris 349 (2011), 21-23.

[ 6 ] M. A. Hill, M. J. Hopkins and D. C. Ravenel, On the non-existence of elements of kervaire invariant one, Ann. of Math. (2) 184 (2016), 1-262.

[ 7 ] N.H.V. Hưng, The weak conjecture on spherical classes, Math. Z. 231 (1999), 727-743.
[ 8 ] M. Kameko, Products of projective spaces as Steenrod modules, PhD. thesis, The Johns Hopkins University, ProQuest LLC, Ann Arbor, MI, 1990, 29 pages.

[ 9 ] W.H. Lin and M. Mahowald, The Adams spectral sequence for Minami's Theorem, in: Proceedings of the Northwestern Homotopy Theory Conference (Providence, Rhode Island) (H. R. Miller and S. B. Priddy, eds.), Contemp. Math. vol. 220, 1983, pp. 143-177.

[10] W.H. Lin, $\operatorname{Ext}_{\mathscr{A}}^{4, *}(\mathbb{Z} / 2, \mathbb{Z} / 2)$ and $\operatorname{Ext}_{\mathscr{A}}^{5, *}(\mathbb{Z} / 2, \mathbb{Z} / 2)$, Topol. Appl. 155 (2008), 459-496.

[11] M. Mahowald, A new infinite family in ${ }_{2} \pi_{*}^{S}$, Topology 16 (1977), 249-256.

[12] N. Minami, The Adams spectral sequence and the triple transfer, Amer. J. Math. 117 (1995), 965985.

[13] N. Minami, The iterated transfer analogue of the new doomsday conjecture, Trans. Amer. Math. Soc. 351 (1999), 2325-2351.

[14] S.A. Mitchell, Splitting $B(\mathbb{Z} / p)^{n}$ and $B T^{n}$ Via Modular Representation Theory, Math. Z. 189 (1985), 1-9.

[15] M.F. Mothebe and L. Uys, Some relations between admissible monomials for the polynomial algebra, Int. J. Math. Math. Sci., Article ID 235806, 2015, 7 pages.

[16] F.P. Peterson, Generators of $H^{*}\left(\mathbb{R} P^{\infty} \times \mathbb{R} P^{\infty}\right)$ as a module over the Steenrod algebra, Abstracts Amer. Math. Soc., Providence, RI, April 1987.

[17] Đ.V. Phúc, The "hit" problem of five variables in the generic degree and its application, Topol. Appl. 282 (2020), 107321, 34 pages.

[18] Đ.V. Phúc, On Peterson's open problem and representations of the general linear groups, J. Korean Math. Soc. 58 (2021), 643-702.

[19] Đ.V. Phúc, Structure of the space of $G L_{4}\left(\mathbb{Z}_{2}\right)$ coinvariants $\mathbb{Z}_{2} \otimes_{G L_{4}\left(\mathbb{Z}_{2}\right)} P H_{*}\left(\mathbb{Z}_{2}^{4}, \mathbb{Z}_{2}\right)$ in some generic degrees and its application, Preprint (2021), submitted for publication.

[20] Đ.V. Phúc, A note on the modular representation on the $\mathbb{Z} / 2$-homology groups of the fourth power of real projective space and its application, Preprint (2021), submitted for publication.

[21] Đ.V. Phúc, On the dimension of $H^{*}\left(\left(\mathbb{Z}_{2}\right)^{\times t}, \mathbb{Z}_{2}\right)$ as a module over Steenrod ring, Topol. Appl. 303 (2021), 107856, 43 pages.

[22] W.M. Singer, The transfer in homological algebra, Math. Z. 202 (1989), 493-523.

[23] W.M. Singer, Rings of symmetric functions as modules over the Steenrod algebra, Algebr. Geom. Topol. 8 (2008), 541-562.

[24] N. Sum, On the Peterson hit problem, Adv. Math. 274 (2015), 432-489.

[25] N. Sum, On the determination of the Singer transfer, Vietnam Journal of Science, Technology and Engineering, 60 (2018), 3-16. 
[26] N. Sum, On a construction for the generators of the polynomial algebra as a module over the Steenrod algebra, in Singh M., Song Y., Wu J. (eds), Algebraic Topology and Related Topics. Trends in Mathematics. Birkhäuser/Springer, Singapore (2019), 265-286.

[27] N.K. Tín, The hit problem for the polynomial algebra in five variables and applications, $\mathrm{PhD}$. thesis, Quy Nhon University, 2017.
[28] G. Walker and R.M.W. Wood, Polynomials and the mod 2 Steenrod Algebra: Volume 1, The Peterson hit problem, in London Math. Soc. Lecture Note Ser., Cambridge Univ. Press, January 11, 2018.

[29] R.M.W. Wood, Steenrod squares of polynomials and the Peterson conjecture, Math. Proc. Cambriges Phil. Soc. 105 (1989), 307-309. 
